\title{
Iterative Learning Control with Time-Partitioned Update for Collaborative Output Tracking
}

\author{
Santosh Devasia \\ Mechanical Engineering Department, U. of Washington, Seattle, USA 98195-2600
}

\begin{abstract}
This article studies iterative learning control (ILC) where multiple heterogeneous linear subsystems (with potentially different individual dynamics) update their input simultaneously based on the error in a collaboratively-controlled desired output. A challenge is that convergence of iterative learning for each individual subsystem (when the other subsystems are not learning) may not guarantee convergence under collaborative-(co-) learning. This work proposes an update-partitioning approach for colearning and demonstrates convergence whenever the individual, iterative learning for each subsystem is convergent. The main contribution of this work is to show that any unity partition (where the sum of the partition is one) of the update law ensures convergence of the co-learning. Since the time partitioning of the update can be chosen independent of the individual learning (convergence) rate of the subsystems, the proposed approach enables the separate design of each individual subsystem's inputupdate law followed by conjoining of the individual update laws for co-learning using the partitioning approach. Additionally, an intermittent time partitioning is developed when the desired trajectory is not known to all (but only some) of the co-learning subsystems.
\end{abstract}

Key words: Iterative learning control; Convergence; Cooperative control; Heterogeneous systems; Output tracking

\section{Introduction}

This article studies iterative learning control (ILC) where multiple $(n)$ heterogeneous linear subsystems (with potentially different individual dynamics, $G_{i}$ ) update their input $u_{i}$ simultaneously based on the error in a collaboratively-controlled output $y$ described in the Laplace domain by $y(s)=\sum_{i=1}^{n} G_{i}(s) u_{i}(s)$. Such collaborative control of a common output $y$ arises in applications such as: (i) active orthosis where a joint is being controlled by a human and a machine, e.g., [1]; (ii) dualstage positioners where a high-bandwidth low-range actuator augments the performance of a low-bandwidth but large-range actuator, e.g., [2,3]. Multiple (redundant) actuators have also been used for collaborative positioning, e.g., using independent actuators or legs to move a single stage, e.g., [4,5], which is similar to use of multiple actuators in nature such as arrays of cilia for movement in fluid environments, multiple muscle fibers in biological actuators, and multiple-legs in centipedes and millepedes. With increasingly distributed sensing, computing and actuation, there is interest in each of the subsystems to operate somewhat independently, which can enable the potential recruitment of different actu-

Email address: devasia@uw.edu (Santosh Devasia). ator subsystems for varying tasks, e.g., similar to the recruitment of different muscle fiber sets (motor units) to manage changing muscle loads. Moreover, since complex tasks could be broken into previously designed trajectories, there is interest in learning to control specific desired (pre-specified) trajectories. Therefore, this work investigates the iterative learning of a common desired output trajectory with multiple subsystems.

Convergence of iterative learning to achieve output tracking of a single output, with a single subsystem, has been well studied, e.g., [6-12]. Note that, for perfect control of a desired output $y=y_{d}$ with a single system $G$, the input $u$ depends on the inverse $G^{-1}$ of the system $G$, i.e., $u=G^{-1} y_{d}$. This has motivated the use of the inverse $\hat{G}^{-1}$ of the known model $\hat{G}$ of the system $G$ in the iteration update law $[7,8,13]$. For such cases, convergence to the desired output can be shown provided the phase error in the model is less than $\pi / 2$ and the iteration gain is sufficiently small [8]. This work aims to generalize such convergence conditions, developed for single input single output systems, to multiple subsystems that collaboratively learn to track a single desired output. However, for iterative co-learning with multiple subsystems, the output error depends on all the $n$ subsystems $\left\{G_{i}\right\}_{i=1}^{n}$, and therefore the update for the input $u_{i}$ for each subsystem (and therefore, the convergence 
condition) becomes dependent on the dynamics of the other subsystems. This interdependence implies that it can be challenging to determine convergence without knowledge of the dynamics of all the subsystems.

The specific issue addressed here is the question wether convergence of iterative learning for each individual subsystem (when the other subsystems are not learning) can be used to develop convergence conditions that do not require knowledge of the dynamics of all the subsystems. Note that convergence properties for special cases are known. For example, convergence follows if only one of the subsystems is actively learning and the others are not. Moreover, since typical convergence conditions constrain the gain of the update law to be sufficiently small, it is also possible to demonstrate that lack of co-ordination (e.g., when all the systems aim to learn simultaneously based on individual update laws) can lead to loss of convergence. For example, the gain of the update law increases and can lead to loss of convergence with increasing number $n$ of (similar) subsystems that are using the same update law. Establishing convergence is also possible when the desired output $y_{d}$ can be mapped into relatively-independent predetermined desired output $y_{d, i}$ for each subsystem, e.g., for manipulation with multiple (but minimal number of) robotic arms [14]. Moreover, convergence can be established for consensus-type algorithms that seek to achieve the same output $y_{i}=y_{d}$ for each agent in networked multi-agent systems, e.g., $[15,16]$ — in contrast, the current work aims to collaboratively control a single total output, $y_{d}=\sum_{i} y_{i}$, where all the individual subsystem outputs $y_{i}$ are not required to be equal.

The main contribution of this work is to show that any unity partition (where the sum of the partition is one) of the update ensures convergence of the co-learning. While the selection of the update partitioning does require some centralization, it could also be as simple as an equal distribution of the update authority to each subsystem, i.e., a scaling of the update by $1 / n$ where $n$ is the number of subsystems, as shown with the simulation example. The specific partition can be frequency dependent and time varying, and therefore, be used to develop algorithms that modify the distribution of control effort among the different subsystems and potentially manage actuator bandwidth constraints in different frequency ranges as in [17]. Additionally, an intermittent time-partitioning-based update law is developed for the case when the desired trajectory is not known to all of the subsystems that are co-learning.

\section{Problem formulation}

The goal is to achieve desired output $y=y_{d}$ for a system with $n$ linear subsystems $\left\{G_{i}\right\}_{i=1}^{n}$, described by

$$
y(s)=\sum_{i=1}^{n} G_{i}(s) u_{i}(s) .
$$

The $n$ inputs to achieve the desired output $y_{d}$ are colearnt in an iterative manner, with the update law described in the frequency domain at the $k^{\text {th }}$ iteration step,

$$
u_{i, k+1}(\omega)=u_{i, k}(\omega)+\rho_{i, k}(\omega) \hat{G}_{i}^{-1}(\omega)\left[y_{d}(\omega)-y_{k}(\omega)\right]
$$

for all frequency $\omega, i=1, \ldots n$, where $\rho_{i, k}(\omega)$ is a realvalued scalar, $k$ is a positive integer, $y_{k}$ is the output,

$$
y_{k}(\omega)=\sum_{i=1}^{n} G_{i}(\omega) u_{i, k}(\omega)
$$

the term in the square bracket in Eq. (2) represents the output error, $\hat{G}_{i}$ is the known model of the $i^{\text {th }}$ subsystem $G_{i}$, and the value of a model $G$ evaluated on the imaginary axis of the complex plane is defined as $G(\omega)=\left.G(s)\right|_{s=j \omega}$ with $j=\sqrt{-1}$.

\section{Assumption 1 (System and model properties)}

In the following, each subsystem $G_{i}(i=1, \ldots n)$ and its model $\hat{G}_{i}$ are stable with hyperbolic zero dynamics, i.e., all zeros have a nonzero real part. Also, these transfer functions are not trivial $G_{i} \neq 0$ and $\hat{G}_{i} \neq 0$.

Remark 1 (Model invertibility) Hyperbolic zero $d y$ namics implies invertibility of the model $\hat{G}_{i}(\omega)$ in the input update law in Eq. (2), and also ensures robustness of the inverse under modeling uncertainty [17].

With a single input $u_{m}$, the iterative control in Eq. (2) converges if the modeling error is sufficiently small, as shown in [8], and stated formally below.

Lemma 1 (Single-input convergence) With only one input $u_{i}$, i.e., $u_{m, 1}=0$ and $\rho_{m, k}=0$, for all $m \neq i$, a finite initial input $u_{i, 1}(\omega)$ and a fixed iteration gain $\bar{\rho}_{i}(\omega)=\rho_{i, k}(\omega)$, the single-input iterations in Eq. (2) converges to the inverse input $u_{i, i n v}$ at frequency $\omega$, i.e., $\lim _{k \rightarrow \infty} u_{i, k}(\omega)=u_{i, i n v}(\omega)=G_{i}^{-1}(\omega)\left[y_{d}(\omega)\right]$, which results in exact tracking of the desired output $y_{d}(\omega)$, i.e., $\lim _{k \rightarrow \infty} y_{k}(\omega)=\lim _{k \rightarrow \infty} G_{i}(\omega) u_{i, k}(\omega)=y_{d}(\omega)$ if and only if the magnitude of the phase uncertainty $\Delta_{\Phi, i}$ in the model and the update gain $\bar{\rho}_{i}(\omega)$ are sufficiently small

$$
\begin{aligned}
& \left|\Delta_{\Phi, i}(\omega)\right|<\pi / 2 \\
& 0<\bar{\rho}_{i}(\omega)<\frac{2 \cos \left[\Delta_{\Phi, i}(\omega)\right]}{\Delta_{M, i}(\omega)}=\rho_{i}^{*}(\omega)
\end{aligned}
$$

where the magnitude uncertainty $\Delta_{M, i}$ and the phase uncertainty $\Delta_{\phi, i}$ are defined by

$$
\frac{G_{i}(\omega)}{\hat{G}_{i}(\omega)}=\Delta_{M, i}(\omega) e^{j \Delta_{\phi, i}(\omega)} .
$$

Proof: This follows from Lemma 1 in [8]. 
Assumption 2 In the following, it is assumed that the modeling uncertainty for each subsystem $G_{i}$ and the associated fixed gain $\bar{\rho}_{i}(\omega) \neq 0$ satisfy the single-input convergence conditions in Eq. (4).

The challenge to establish convergence with co-learning is the interdependence of the subsystems in the update law. In particular, the input update can be rewritten from Eqs. (1), (2) as

$$
\begin{aligned}
u_{i, k+1}(\omega)-u_{i, k}(\omega) & \\
= & \left(1-\frac{\rho_{i, k}(\omega) G_{i}(\omega)}{\hat{G}_{i}(\omega)}\right)\left[u_{i, k}(\omega)-u_{i, k-1}(\omega)\right] \\
& -\sum_{j=1, j \neq i}^{n} \frac{\rho_{i, k}(\omega) G_{j}(\omega)}{\hat{G}_{i}(\omega)}\left[u_{j, k}(\omega)-u_{j, k-1}(\omega)\right] .
\end{aligned}
$$

Without the second term on the right hand side, the input-learning iterations would be a contraction if the gain term $1-\left(\rho_{i, k}(\omega) G_{i}(\omega) / \hat{G}_{i}(\omega)\right)$ is small. However, the interdependence of the subsystems due to the coupling between the model $\hat{G}_{i}$ and the other subsystems $G_{j}$, in the second term, makes it challenging to develop conditions for co-learning. Hence, the problem is to establish convergence conditions for distributed co-learning with multiple inputs as in Eq. (2) when the single-input convergence conditions in Eq. (4) are met as, in Assumption 2.

\section{Convergence conditions}

It is shown that single-input convergence conditions can be used to develop co-learning convergence by partitioning the iterative update. The partition is defined below.

Definition 1 (Time-partition) A time partition $\mathcal{P}$ is a set of partitions $\mathcal{P}_{k}$ at each iteration step $k$

$$
\mathcal{P}(\omega)=\left\{\mathcal{P}_{k, n}(\omega)\right\}_{k=1,2, \ldots}^{\infty}
$$

where the partition $\mathcal{P}_{k, n}(\omega)$ consists of $n$ terms $\mathcal{P}_{k, n}(\omega)=\left\{p_{i, k}(\omega)\right\}_{i=1}^{n}$ that are non-negative real-valued scalars $p_{i, k}$, which add to one, i.e.,

$$
0 \leq p_{i, k}(\omega) \leq 1, \quad \sum_{i=1}^{n} p_{i, k}(\omega)=1
$$

Definition 2 (Co-learning with partition) Given a time partition $\mathcal{P}$ (as in Definition 1) and a set of singleinput iteration gains $\left\{\bar{\rho}_{i}\right\}_{i=1}^{n}$, co-learning with partition is defined as the input law in Eq. (2) with the iteration gain $\rho_{i, k}$

$$
\rho_{i, k}(\omega)=\bar{\rho}_{i}(\omega) p_{i, k}(\omega) .
$$

\subsection{Convergence with partitioning}

The following lemma shows that any partition $\mathcal{P}$ of the single-input iteration gain $\bar{\rho}_{i}$ (as in Definition 2) leads to convergence of the corresponding iterative co-learning.
Lemma 2 (Convergence with partition) Given a time partition $\mathcal{P}$ as in Definition 1 , the iterative colearning in Definition 2 converges, i.e., the inputs are finite, $\lim _{k \rightarrow \infty} u_{i, k}(\omega)=u_{i}(\omega)<\infty$ for each $i$, and the output $y_{k}(\omega)$ tends to the desired output $y_{d}(\omega)$

$$
\lim _{k \rightarrow \infty} y_{k}(\omega)=y_{d}(\omega)
$$

point-wise in frequency $\omega$ if the single-input-convergence conditions in Eq. (4) are met for each subsystem $G_{i}$ with iteration gain $\rho_{i, k}(\omega)=\bar{\rho}_{i}(\omega)$, and if the initial inputs $\left\{u_{i, 1}(\omega)\right\}_{i=1}^{n}$, and therefore, the initial output $y_{i, 1}(\omega)=$ $G_{i}(\omega) u_{i, 1}(\omega)$ of each subsystem $i$, are finite.

Proof: Multiplying the iteration-update law for input $u_{i}$ in Eq. (2) by the subsystem $G_{i}$ and then summing over all inputs yield, from Eq. (3)

$$
y_{k+1}(\omega)=y_{k}(\omega)+\sum_{i=1}^{n} \frac{\rho_{i, k}(\omega) G_{i}(\omega)}{\hat{G}_{i}(\omega)}\left[y_{d}(\omega)-y_{k}(\omega)\right]
$$

Subtracting the desired output $y_{d}$ from both sides of this expression results in

$$
\left[y_{k+1}(\omega)-y_{d}(\omega)\right]=\gamma_{k}(\omega)\left[y_{k}(\omega)-y_{d}(\omega)\right]
$$

where the overall gain $\gamma_{k}$

$$
\gamma_{k}(\omega)=1-\sum_{i=1}^{n} \frac{\rho_{i, k}(\omega) G_{i}(\omega)}{\hat{G}_{i}(\omega)}
$$

which can be rewritten from Eqs. (7) and (8), as

$$
\begin{aligned}
\gamma_{k}(\omega) & =\sum_{i=1}^{n} p_{i, k}(\omega)\left[1-\frac{\bar{\rho}_{i}(\omega) G_{i}(\omega)}{\hat{G}_{i}(\omega)}\right] \\
& =\sum_{i=1}^{n} p_{i, k}(\omega) W_{i}(\omega) .
\end{aligned}
$$

The magnitude of the weight $W_{i}$, rewritten using the uncertainty definition in Eq. (5) as

$$
W_{i}(\omega)=\left[1-\bar{\rho}_{i}(\omega) \Delta_{M, i}(\omega) e^{j \Delta_{\phi, i}(\omega)}\right]
$$

is strictly smaller than one, if and only if its square is strictly smaller than one, which is given by

$$
\begin{aligned}
\left|W_{i}(\omega)\right|^{2}= & \left|1-\bar{\rho}_{i}(\omega)\left[\Delta_{M, i}(\omega)\right] e^{j \Delta_{\Phi, i}(\omega)}\right|^{2} \\
= & 1+\bar{\rho}_{i}^{2}(\omega)\left[\Delta_{M, i}(\omega)\right]^{2} \\
& -2 \bar{\rho}_{i}(\omega)\left[\Delta_{M, i}(\omega)\right] \cos \left(\Delta_{\Phi, i}(\omega)\right) \\
= & 1+\bar{\rho}_{i}(\omega)\left[\Delta_{M, i}(\omega)\right]^{2}\left\{\bar{\rho}_{i}(\omega)-\frac{2 \cos \left(\Delta_{\Phi, i}(\omega)\right)}{\Delta_{M, i}(\omega)}\right\}
\end{aligned}
$$

The magnitude ratio $\Delta_{M, i}(\omega)$ is nonzero, i.e., $\Delta_{M, i}(\omega)=$ $\left|G_{i}(\omega)\right| /\left|\hat{G}_{i}(\omega)\right|>0$ since the model $\hat{G}_{i}$ is assumed to be stable $\left|\hat{G}_{i}(\omega)\right| \neq \infty$ and the system $G_{m}$ is assumed 
to have hyperbolic zero-dynamics $\left|G_{i}(\omega)\right| \neq 0$ under Assumption 1. From the single-input-convergence conditions in Eq. (4), the iteration gain is nonzero, i.e., $\bar{\rho}_{i}(\omega) \neq 0$, and the term in the curly brackets in Eq. (15) is negative resulting in $0 \leq\left|W_{i}(\omega)\right|^{2}<1$, which in turn results in the magnitude of weight $W_{i}$ being strictly smaller than one, i.e., $0 \leq\left|W_{i}(\omega)\right|<1$. Moreover, the maximum value $\bar{W}$ of the weights $W_{i}$ is also strictly less than one,

$$
0 \leq\left[\bar{W}(\omega)=\max _{i=1,2, \ldots, n}\left|W_{i}(\omega)\right|\right]<1
$$

which implies that the magnitude of the overall iteration gain $\gamma_{k}$ is also less than one, i.e., from Eqs. (7) and (13),

$$
\begin{aligned}
\left|\gamma_{k}(\omega)\right| & \leq \sum_{i=1}^{n}\left|p_{i, k}(\omega) W_{i}(\omega)\right| \\
& \leq \bar{W}(\omega) \sum_{i=1}^{n} p_{i, k}(\omega)=\bar{W}(\omega)<1
\end{aligned}
$$

Therefore, the sequence of outputs $y_{k}(\omega)$ in Eq. (11) is converging with

$$
\begin{aligned}
\left|y_{k+1}(\omega)-y_{d}(\omega)\right| & \leq \bar{W}(\omega)\left|y_{k}(\omega)-y_{d}(\omega)\right| \\
& \leq[\bar{W}(\omega)]^{k}\left|y_{1}(\omega)-y_{d}(\omega)\right|
\end{aligned}
$$

which converges since maximum magnitude of the weights $\bar{W}$ is less than one from Eq. (16). The finiteness of the input can be established from the update law in Eq. (2) and from Eqs. (7), (8)

$$
\begin{aligned}
\left|u_{i, k+1}(\omega)\right| \leq & \left|u_{i, k}(\omega)\right|+\left|\bar{\rho}_{i} \hat{G}_{i}^{-1}(\omega)\right|\left|y_{d}(\omega)-y_{k}(\omega)\right| \\
\leq & \left|u_{i, 1}(\omega)\right| \\
& +\left|\bar{\rho}_{i} \hat{G}_{i}^{-1}(\omega)\right| \sum_{m=1}^{k}\left|y_{d}(\omega)-y_{m}(\omega)\right| \\
& \text { from Eq. }(2) \\
\leq & \left|u_{i, 1}(\omega)\right| \\
& +\left|\bar{\rho}_{i} \hat{G}_{i}^{-1}(\omega)\right|\left|y_{d}(\omega)-y_{1}(\omega)\right| \sum_{m=1}^{k}[\bar{W}(\omega)]^{m-1} \\
& \quad \text { from Eq. }(18) \\
\leq & \left|u_{i, 1}(\omega)\right|+\frac{\left|\bar{\rho}_{i} \hat{G}_{i}^{-1}(\omega)\right|}{1-\bar{W}(\omega)}\left|y_{d}(\omega)-y_{1}(\omega)\right| .
\end{aligned}
$$

This completes the proof since maximum magnitude of the weights, $\bar{W}$, is less than one from Eq. (16).

Remark 2 (Partitions whose sum is less than one) A time partition, similar to that in Eq. (7), but whose sum adds to a fixed value $p^{*}(\omega)$ that is less than one, i.e.,

$$
0 \leq p_{i, k}(\omega) \leq 1, \quad 0<\sum_{i=1}^{n} p_{i, k}(\omega)=p^{*}(\omega) \leq 1
$$

will also lead to convergence at frequency $\omega$, as in Lemma 2. This can be established from Lemma 2 by considering a modified (smaller) iteration gain $\bar{\rho}_{i}^{*}(\omega)$ and a modified partition $p_{i, k}^{*}(\omega)$ (that adds to one) given by

$$
\bar{\rho}_{i}^{*}(\omega)=\bar{\rho}_{i}(\omega) p^{*}(\omega) \text { and } p_{i, k}^{*}(\omega)=p_{i, k}(\omega) / p^{*}(\omega)
$$

\subsection{Noise effects}

If noise is present in the output at iteration step $k$, then the noise effects can potentially build up with iterations. Noise is represented by a term $N_{k}$ that modifies the output at the $k^{\text {th }}$ iteration in Eq. (3) to

$$
y_{k}(\omega)=N_{k}(\omega)+\sum_{i=1}^{n} G_{i}(\omega) u_{i, k}(\omega)
$$

The effect of the noise is bounded if the noise is bounded as shown below for the distributed co-learning case based on a similar proof in [8] for the single input case.

Lemma 3 (Noise effect) Under conditions of Lemma 2, if the output noise $N_{k}$ in Eq. (22) is bounded as

$$
\left|N_{k}(\omega)\right| \leq \bar{N}(\omega)
$$

where $\bar{N}(\omega)$ is a real-valued scalar, then the error in the output with the iteration update law in Eq. (2) due to the noise is bounded. In particular, given any arbitrarily small positive constant $\epsilon_{N}>0$, there exists an integer $k_{N}(\omega)$ such that

$$
\left|y_{k}(\omega)-y_{d}(\omega)\right| \leq \frac{2 \bar{N}(\omega)}{1-\bar{W}(\omega)}+\epsilon_{N} \quad \forall k>k_{N}(\omega)
$$

where $\bar{W}$ is the upper bound on the magnitude of the overall iteration gain $\gamma_{k}$ from Eq. (17).

Proof: Multiplying the iteration-update law for input $u_{i}$ in Eq. (2) by the subsystem $G_{i}$, summing over all inputs as in Eq. (3), and using the definition of the overall gain $\gamma_{k}$ in Eq. (12), yields the following expression for the output $y_{k+1}$, which is similar to Eq. (10),

$$
\begin{aligned}
y_{k+1}(\omega)= & y_{k}(\omega)+\left(1-\gamma_{k}(\omega)\right)\left[y_{d}(\omega)-y_{k}(\omega)\right] \\
& +\left[N_{k+1}(\omega)-N_{k}(\omega)\right] .
\end{aligned}
$$

Subtracting the desired output $y_{d}$ from both sides of this expression, using the bound $\bar{W}$ on the overall iteration gain $\gamma_{k}$ in Eq. (17), and replacing the the noise $N$ with its bound $\bar{N}$ in Eq. (23), leads to

$$
\begin{aligned}
\left|y_{k+1}(\omega)-y_{d}(\omega)\right| \leq & |\bar{W}(\omega)|^{k}\left|y_{1}(\omega)-y_{d}(\omega)\right| \\
& +2|\bar{N}(\omega)| \sum_{m=1}^{k}[\bar{W}(\omega)]^{m-1} \\
\leq & |\bar{W}(\omega)|^{k}\left|y_{1}(\omega)-y_{d}(\omega)\right|+\frac{2|\bar{N}(\omega)|}{1-\bar{W}(\omega)}
\end{aligned}
$$

and the Lemma follows since the maximum magnitude of the weights, $\bar{W}$, is less than one from Eq. (16). 


\section{Restricted access to desired output}

Consider an augmented system as defined below where the new $(n+1)^{t h}$ subsystem does not have access to the desired output $y_{d}$ (say due to communication constraints) but has access to its own output $y_{n+1, k}$ and the total output $y_{k}$ at each iteration step, where

$$
y_{n+1, k}(\omega)=G_{n+1}(\omega) u_{n+1, k}(\omega) \text {. }
$$

Definition 3 (Restricted access system) The system with access restrictions has $n+1$ subsystems, i.e., Eq. (1) becomes

$$
y(s)=\sum_{i=1}^{n+1} G_{i}(s) u_{i}(s),
$$

where the original system with $n$ subsystems in Eq. (1) satisfies the co-learning convergence conditions in Lemma 2 for a partition $\mathcal{P}$ as in Definition 1 where the maximum $\bar{W}$ in Eq. (16) of the iteration weights $\left\{\bar{W}_{i}\right\}_{i=1}^{n}$, defined in Eq. (14) is less than one. Moreover, it is assumed that the new subsystem $G_{n+1}$

(1) satisfies the single-input-convergence conditions in $E q$. (4) for the constant iteration gain $\rho_{n+1, k}(\omega)=$ $\bar{\rho}_{n+1}(\omega)$, and

(2) its initial input $\left\{u_{n+1,1}(\omega)\right\}$ is finite.

Definition 4 (Modified update) The iteration update for the restricted-access subsystem in Definition 3 can be modified to use the system output $y_{k}$ at iteration step $k$, instead of the desired output $y_{d}$ in Eq. (2), i.e.,

$$
\begin{aligned}
u_{n+1, k+1}(\omega)= & u_{n+1, k}(\omega) \\
& +\rho_{n+1, k} \hat{G}_{n+1}^{-1}(\omega)\left[\beta_{n+1} y_{k}(\omega)-y_{n+1, k}(\omega)\right] \\
= & u_{n+1, k}(\omega) \\
& +\rho_{n+1, k} \hat{G}_{n+1}^{-1}(\omega) \beta_{n+1}\left[y_{k}(\omega)-y_{d}(\omega)\right] \\
& +\rho_{n+1, k} \hat{G}_{n+1}^{-1}(\omega)\left[\beta_{n+1} y_{d}(\omega)-y_{n+1, k}(\omega)\right]
\end{aligned}
$$

with a real-valued scalar, $0 \leq \beta_{n+1} \leq 1$, and the iteration gain $\rho_{n+1, k}$ is given by

$$
\rho_{n+1, k}(\omega)=\bar{\rho}_{n+1}(\omega) p_{n+1, k}(\omega)
$$

The goal is to design the iteration gain $\rho_{n+1, k}$ in Eq. (30) to ensure convergence of this modified learning approach. In the following, it is shown that convergence can be achieved if the update of the new $(n+1)^{t h}$ subsystem is intermittent.

Definition 5 (Intermittent update) The update of the new $(n+1)^{\text {th }}$ subsystem is intermittent, i.e.,

$$
\begin{aligned}
p_{n+1, k} & =1 & & \text { iff } k \bmod M=0 \\
& =0 & & \text { otherwise }
\end{aligned}
$$

where $k$ and the constant $M$ are positive integers. Note that the iteration gain $\rho_{n+1, k}$ in Eq. (30) is zero for $M-1$ iteration steps between the updates.

\section{Lemma 4 (Intermittent-update convergence)}

Let the update of the new $(n+1)^{\text {th }}$ subsystem be intermittent as in Definition 5. Then, the iterative co-learning with restricted access to the output as in Definition 4 converges, i.e., the inputs are finite, $\lim _{k \rightarrow \infty} u_{i, k}(\omega)=u_{i}(\omega)<\infty$ for $1 \leq i \leq n+1$, the output $y_{k}$ tends to the desired output $y_{d}$, and the output $y_{n+1, k}$ of the new system converges to $\beta_{n+1} y_{d}$,

$$
\begin{aligned}
\lim _{k \rightarrow \infty} y_{k}(\omega) & =y_{d}(\omega) \\
\lim _{k \rightarrow \infty} y_{n+1, k}(\omega) & =\beta_{n+1} y_{d}(\omega)
\end{aligned}
$$

point-wise in frequency $\omega$, if the iteration gain $\rho_{n+1}$ in Eq. (30) is sufficiently small, i.e, chosen to satisfy

$$
\bar{\rho}_{n+1}(\omega)<\frac{\cos \left[\Delta_{\Phi, n+1}(\omega)\right]}{\Delta_{M, n+1}(\omega)}
$$

and the number of steps $M$ between the intermittent updates in Eq. (31) is sufficiently large.

Proof: Let the error in the output $y_{k}$ and in the output of the new system $y_{n+1, k}$ at each iteration step $k \geq 1$ be

$$
\begin{aligned}
e_{k}(\omega) & =y_{d}(\omega)-y_{k}(\omega) \\
e_{n+1, k}(\omega) & =\beta_{n+1} y_{d}(\omega)-y_{n+1, k}(\omega) .
\end{aligned}
$$

Expressions for these errors can be found by multiplying the iteration-update law for input $u_{i}$ in Eqs. (2), (29) by the subsystem $G_{i}$, for $i=1 \ldots(n+1)$, and using Definition 4, and Eqs. (13), (27), (31), for all positive integers $k$ such that $k \bmod M=0$,

$$
\begin{aligned}
e_{k+1}(\omega)= & e_{k}(\omega) \\
& -\sum_{i=1}^{n} \frac{\rho_{i, k}(\omega) G_{i}(\omega)}{\hat{G}_{i}(\omega)}\left[y_{d}(\omega)-y_{k}(\omega)\right] \\
& -\frac{\bar{\rho}_{n+1}(\omega) G_{n+1}(\omega)}{\hat{G}_{n+1}(\omega)}\left[\beta_{n+1} y_{k}(\omega)-y_{n+1, k}(\omega)\right] \\
= & \gamma_{k}(\omega) e_{k}(\omega) \\
& -\beta_{n+1} \frac{\bar{\rho}_{n+1}(\omega) G_{n+1}(\omega)}{\hat{G}_{n+1}(\omega)}\left[y_{k}(\omega)-y_{d}(\omega)\right] \\
& -\frac{\bar{\rho}_{n+1}(\omega) G_{n+1}(\omega)}{\hat{G}_{n+1}(\omega)}\left[\beta_{n+1} y_{d}(\omega)-y_{n+1, k}(\omega)\right] \\
= & \gamma_{k}(\omega) e_{k}(\omega) \\
& +\beta_{n+1}\left[1-W_{n+1}(\omega)\right] e_{k}(\omega) \\
& -\left[1-W_{n+1}(\omega)\right] e_{n+1, k}(\omega), \\
e_{n+1, k+1}(\omega)= & e_{n+1, k}(\omega) \\
& +\beta_{n+1} \frac{\bar{\rho}_{n+1}(\omega) G_{n+1}(\omega)}{\hat{G}_{n+1}(\omega)}\left[e_{k}(\omega)\right] \\
& -\frac{\bar{\rho}_{n+1}(\omega) G_{n+1}(\omega)}{\hat{G}_{n+1}(\omega)}\left[e_{n+1, k}(\omega)\right] \\
= & W_{n+1}(\omega) e_{n+1, k}(\omega) \\
& +\beta_{n+1}\left[1-W_{n+1}(\omega)\right] e_{k}(\omega) .
\end{aligned}
$$


If the positive integer $k$ does not satisfy $k \bmod M=0$, then the new subsystem input $u_{n+1, k+1}$ is not updated, i.e., similar to Eqs. (35), (36),

$$
\begin{aligned}
e_{k+1}(\omega) & =\gamma_{k}(\omega) e_{k}(\omega) \\
e_{n+1, k+1}(\omega) & =e_{n+1, k}(\omega)
\end{aligned}
$$

From Eqs. (35)-(37) the change in the error $E$

$$
E[K]=\left[\begin{array}{c}
e_{((K-1) M+1)} \\
e_{(n+1,(K-1) M+1)}
\end{array}\right]
$$

over $M$ iteration steps can be related as

$$
E[K+1]=\mathcal{M}_{M} E[K]
$$

where, for all positive integers $K$,

$$
\begin{aligned}
\mathcal{M}_{M}= & {\left[\begin{array}{rl}
\gamma_{k}(\omega)+\beta_{n+1}\left[1-W_{n+1}(\omega)\right] & -\left[1-W_{n+1}(\omega)\right] \\
+\beta_{n+1}\left[1-W_{n+1}(\omega)\right] & W_{n+1}(\omega)
\end{array}\right] } \\
& \times\left[\begin{array}{rr}
{\left[\gamma_{k}(\omega)\right]^{M-1}} & 0 \\
0 & 1
\end{array}\right] .
\end{aligned}
$$

Let the effect of intermittent update be represented by the parameter $\epsilon$

$$
\epsilon(M)=\left[\gamma_{k}(\omega)\right]^{M-1} .
$$

Note that in the limit when the number of iteration steps $M$ between updates of the new system tends towards infinity, the term $\epsilon=0$, and the matrix $\mathcal{M}_{M}$ in Eq. (40) becomes

$$
\left.\mathcal{M}_{M}\right|_{\epsilon=0}=\left[\begin{array}{ll}
0 & -\left[1-W_{n+1}(\omega)\right] \\
0 & W_{n+1}(\omega)
\end{array}\right] .
$$

The singular values of matrix $\mathcal{M}_{M}(0)$ are the roots of the eigenvalues of the matrix

where

$$
\mathcal{M}_{M}(0)^{*} \mathcal{M}_{M}(0)=\left[\begin{array}{ll}
0 & 0 \\
0 & \sigma_{M}^{2}(\omega)
\end{array}\right]
$$

$$
\begin{aligned}
& \sigma_{M}^{2}(\omega)=[1-\left.W_{n+1}^{*}(\omega)\right]\left[1-W_{n+1}(\omega)\right] \\
&+W_{n+1}^{*}(\omega) W_{n+1}(\omega) \\
&= \bar{\rho}_{n+1}^{2}(\omega) \Delta_{M, n+1}^{2}(\omega) \\
&+\left[1-\bar{\rho}_{n+1}(\omega) \Delta_{M, n+1}(\omega) e^{-j \Delta_{\phi, n+1}(\omega)}\right] \\
& \times\left[1-\bar{\rho}_{n+1}(\omega) \Delta_{M, n+1}(\omega) e^{j \Delta_{\phi, n+1}(\omega)}\right] \\
& \text { from Eq. }(14) \\
&=1+ \\
& \quad \times \bar{\rho}_{n+1}(\omega) \Delta_{M, n+1}^{2}(\omega) \\
& \times\left\{\bar{\rho}_{n+1}(\omega)-\frac{\cos \left[\Delta_{\Phi, n+1}(\omega)\right]}{\Delta_{M, n+1}(\omega)}\right\} .
\end{aligned}
$$

Hence both the singular values $\left\{0, \sigma_{M}\right\}$ of the matrix $\mathcal{M}_{M}(0)$ are less than one since $\sigma_{M}^{2}(\omega)<1$ if the iteration gain for the new $(n+1)^{t h}$ subsystem is sufficiently small to satisfy Eq. (33) of the Lemma. Due to continuity of singular values with parameters, the singular values of $\mathcal{M}_{M}$ will remain close to the singular values of $\mathcal{M}_{M}(0)$ (when $\epsilon(M)=0$ ) provided the term $\epsilon(M)$ remains small. Due to exponential dependence of the number of iteration steps $M$ between intermittent updates of the new system and $\epsilon(M)$ from Eq. (41), the singular values of $\mathcal{M}_{M}$ are less than one if the number of steps $M$ between intermittent updates is sufficiently large. Convergence of the output follows since $\sigma_{M}<1$, and from Eq. (39)

$$
\|E[K+1]\|_{2} \leq\left\|\mathcal{M}_{M}\right\|_{2}^{K}\|E[1]\|_{2} \leq \sigma_{M}^{K}\|E[1]\|_{2}
$$

where $\|\cdot\|_{2}$ represents the Euclidean two norm of vectors, and the induced-two norm (square root of the maximum singular value) for matrices. Finiteness of the inputs $\left\{u_{i}(\omega)\right\}_{i=1}^{n}$ follows from arguments similar to those in Eq. (19) in the proof of Lemma 2. Finiteness of the new input $u_{n+1}(\omega)$ also follows from a similar argument, e.g., from Eq. (29) for all $K \geq 1$

$$
\begin{aligned}
\left\|u_{n+1, K M+1}(\omega)\right\|_{2} \leq & \left\|u_{n+1,(K-1) M+1}(\omega)\right\|_{2} \\
& +\left\|\bar{\rho}_{n+1}(\omega) \hat{G}_{n+1}^{-1}(\omega)\right\|_{2} \\
& \times\left\|\left[\begin{array}{ll}
\beta_{n+1} & 1
\end{array}\right]\left[\begin{array}{c}
e_{(K M)} \\
e_{(n+1, K M)}
\end{array}\right]\right\|_{2} \\
\leq & \left\|u_{n+1,(K-1) M+1}(\omega)\right\|_{2}+K^{*} \sigma_{M}^{K-1} \\
& \text { from Eqs. }(37),(38),(45)
\end{aligned}
$$

where

$$
\begin{gathered}
K^{*}=\left\|\bar{\rho}_{n+1}(\omega) \hat{G}_{n+1}^{-1}(\omega)\right\|_{2} \times \\
\left\|\left[\beta_{n+1} \gamma_{k}^{M-1}(\omega) 1\right]\right\|_{2} .
\end{gathered}
$$

The boundedness of the input $u_{n+1}(\omega)$ follows since $\sigma_{M}<1$ and the finiteness of the initial input in Definition 3, since, from Eq. (46)

$$
\begin{aligned}
\left|u_{n+1, K M+1}(\omega)\right| & \leq\left|u_{n+1,1}(\omega)\right|+\sum_{m=1}^{K} K^{*} \sigma_{M}^{m-1} \\
& \leq\left|u_{n+1,1}(\omega)\right|+K^{*} \frac{1}{1-\sigma_{M}} .
\end{aligned}
$$

This completes the proof.

\section{Illustrative example}

Example system: Consider a simplified model of a multiple actuator system that aims to move a mass $M$ using $n$ actuators, each with dynamics $G_{i}$ and generating an individual force $F_{i}=y_{i}$ resulting in a net output force $F$ (output $y$ ) given by

$$
F(s)=\sum_{i=1}^{n} f_{i}(s)=\sum_{i=1}^{n} y_{i}(s)=\sum_{i=1}^{n} G_{i}(s) u_{i}(s),
$$


with subsystem $i$ input $u_{i}$. While more complex actuator dynamics $G_{i}$ could be considered, for simplicity of this illustrative example, each actuator dynamics is assumed to be a first order, of the form

$$
G_{i}(s)=\frac{\tau_{i}}{\tau_{i} s+1}, \quad \tau_{i}=1-\frac{0.5}{i}, \quad i=1, \ldots n .
$$

Single subsystem convergence: Let each agent have the same model $\hat{G}_{i}$ of its dynamics $G_{i}$,

$$
\hat{G}_{i}(s)=\frac{1}{s+1} \quad i=1, \ldots n
$$

Then, the modeling uncertainty, as in Eq. (5), is given by

$$
\Delta_{M, i}(\omega) e^{j \Delta_{\phi, i}(\omega)}=\frac{G_{i}(\omega)}{\hat{G}_{i}(\omega)}=\frac{\left(\alpha_{i} \tau_{i} j \omega+1\right)}{\alpha_{i}\left(\tau_{i} j \omega+1\right)},
$$

with $\alpha_{i}=1 / \tau_{i}$, which is in the form of a standard lead compensator (e.g., see Eq. 10.8 in [18]), and is therefore, bounded by (for all frequency $\omega$ )

$$
\Delta_{M, i}(\omega)<1, \quad\left|\Delta_{\phi, i}(\omega)\right| \leq \cos ^{-1}\left(\frac{2 \sqrt{\alpha_{i}}}{1+\alpha_{i}}\right) .
$$

For the single-input case, with only one actuator $i$, given a desired force output $F_{d}=y_{d}$, the actuator could iteratively learn the needed input (using Eq. (7)) by choosing a sufficiently small iteration gain $\rho_{i}(\omega)$, e.g.,

$$
\rho_{i}(\omega)=\left(\frac{1}{5}\right) \frac{4 \sqrt{\alpha_{i}}}{1+\alpha_{i}}<\frac{2 \cos \left[\Delta_{\Phi, i}(\omega)\right]}{\Delta_{M, i}(\omega)},
$$

which, from Eq. (51) satisfies the single-input convergence conditions in Eq. (4) of Lemma 1.

Co-convergence: Without the time partitioning, i.e., $p_{i, k}(\omega)=1$ in Eq. (8), iterations with the update law in Eq. (7) do not converge as the number $n$ of subsystems increase, e.g., for $n=10$ subsystems, as shown in Fig. 1. However, with knowledge of the number $(n=10)$ of subsystems, a uniform partition, $p_{i, k}(\omega)=1 / n$ in Eq. (8), enables convergence of co-learning as shown in Fig. 1.

Although the proposed approach does not directly handle constraints, the time-and-frequency-dependent partition $\mathcal{P}$ can be designed to adjust the input size of the different subsystems. To illustrate, consider the following selection of the weights that vary inversely with the input magnitude at the previous iteration,

$$
p_{i, k+1}(\omega)=\frac{e^{-\delta \max _{t}\left|u_{i, k}(t)\right|}}{\sum_{i=1}^{n} e^{-\delta \max _{t}\left|u_{i, k}(t)\right|}}
$$

for positive integers $k$ and $\delta=25$ (for this example). The input-equalizing partition in Eq. (53) leads to a relatively-more uniform distribution of the input magnitudes when compared to the uniform partition case $p_{i, k+1}(\omega)=1 / n$, as shown in Fig. 2 . It is noted that other partitions can also lead to similar equalization of the input magnitudes. However, such equalizing partitions require more information such as the magnitudes of all the subsystem inputs $u_{i, k}$ from the previous iteration, and in this sense they require increased co-ordination and communication between the subsystems. Nevertheless, the dynamics and uncertainties of each subsystem (which do affect the individual subsystem's convergence rate) are not necessary to ensure convergence of co-learning using the time partitioning approach. Overall, the results illustrate that the partitioning approach ensures convergence for co-learning.
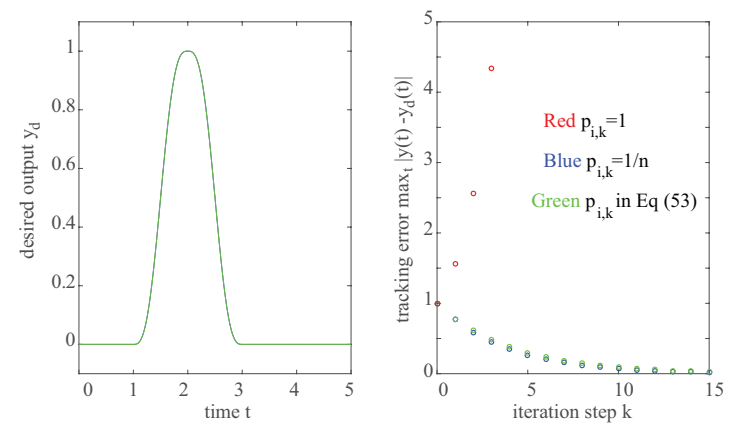

Fig. 1. (Left) Desired output $y_{d}$. (Right) Output tracking error for different choice of update partition $p_{i, k}$
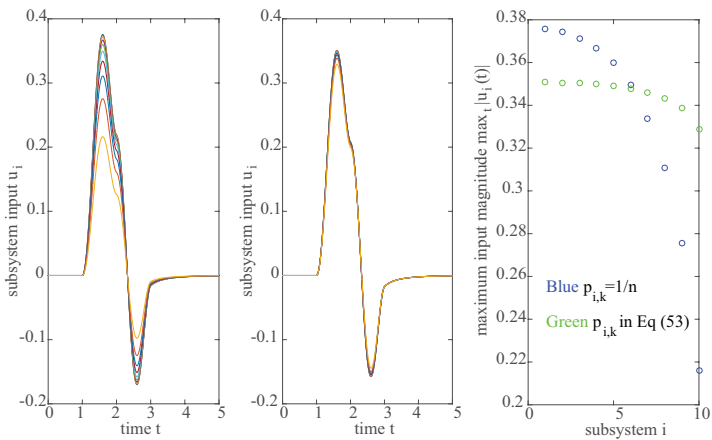

Fig. 2. Subsystem inputs $u_{i}$ for: (left) uniform partition $p_{i, k}=1 / n$; and (middle) input-equalizing partition in Eq. (53). (Right) Maximum magnitude of each subsystem input $u_{i}$ for different choice of update partition $p_{i, k}$.

\section{Conclusion}

The article developed an update-partitioning approach that guarantees convergence of iterative co-learning whenever the individual, iterative learning for each linear subsystem converged. The main contribution was to show that any unity partition (where the sum of the partition is one) of the update law ensures co-learning convergence. The proposed approach enabled the separate design of each individual subsystem's input-update law followed by conjoining for co-learning using the 
update-partitioning approach. Another concept developed in this work was an intermittent update scheme for the case when the desired output was not known to all subsystems.

\section{Acknowledgements}

This work was partially supported by the National Science Foundation (NSF) through grants CMII 1301452 and CMII 1536306.

\section{References}

[1] M Bruce Wiggin, Gregory S Sawicki, and Steven H Collins. An exoskeleton using controlled energy storage and release to aid ankle propulsion. In Rehabilitation Robotics (ICORR), 2011 IEEE International Conference on, pages 1-5. IEEE, 2011.

[2] Steven J. Schroeck, William C. Messner, and Robert J. McNab. On compensator design for linear timeinvariant dual-input single-output systems. IEEE/ASME Transactions on Mechatronics, 6(1):50-57, March 2001.

[3] R. Brinkerhoff and S. Devasia. Output tracking for actuator deficient/redundant systems: Multiple piezoactuator example. AIAA J. of Guidance, Control, and Dynamics, 23(2):370-373, March-April 2000

[4] Won-jong Kim and Yi-chu Chang. Aquatic ionic-polymermetal-composite insectile robot with multi-DOF legs. IEEE/ASME Trans. on Mechatronics, 18(2):547-555, April, 2013.

[5] S. Wilcox and S. Devasia. Stability of velocity control for a piezoelectric stepper. IEEE/ASME Transactions on Mechatronics, 20(2):910-923, April, 2015.

[6] S. Arimoto, S. Kawamura, and F. Miyazaki. Bettering operation of robots by learning. J. of Robotic Systems, 1(2):123-140, March 1984.

[7] J. Ghosh and B. Paden. A pseudo-inverse based iterative learning control. IEEE Trans. on Automatic Control, 47(5):831-837, May,2002.

[8] S. Tien, Q. Zou, and S. Devasia. Iterative control of dynamicscoupling-caused errors in piezoscanners during high-speed AFM operation. IEEE Transactions on Control Systems Technology, 13(6):921-931, Nov 2005.

[9] H.-S. Ahn, Y. Q. Chen, and K. L. Moore. Iterative learning control: Brief survey and categorization. IEEE Transactions on Systems, Man, and Cybernetics, Part C: Applications and Reviews, 37(6):1099-1121, 2007.

[10] J. Ghosh and B. Paden. Iterative learning control for nonlinear nonminimum phase plants. ASME Journal of Dynamic Systems, Measurement, and Control, 123:21-20, March,2001.

[11] S. Mishra and M. Tomizuka. An optimization-based approach for design of iterative learning controllers with accelerated rates of convergence. Proceedings of the 44 th IEEE Conference on Decision and Control,Seville, Spain, 115:2427-2432, December 12-15, 2005.

[12] D.A. Bristow and A.G. Alleyne. Monotonic convergence of iterative learning control for uncertain systems using a timevarying filter. IEEE Transactions on Automatic Control, 53(2):582 - 585, March, 2008.
[13] C. G. Atkeson and J. McIntyre. Robot trajectory learning through practice. In IEEE Int. Conf. on Robotics and Automation, pages 1737-1742, 1986.

[14] H Kawasaki, S Ueki, and S Ito. Decentralized adaptive coordinated control of multiple robot arms without using a force sensor. Automatica, 42(3):481-488, Mar 2006.

[15] Jongeun Choi, Songhwai Oh, and Roberto Horowitz. Distributed learning and cooperative control for multi-agent systems. Automatica, 45(12):2802-2814, DEC 2009.

[16] Weisheng Chen, Shaoyong Hua, and Shuzhi Sam Ge. Consensus-based distributed cooperative learning control for a group of discrete-time nonlinear multi-agent systems using neural networks. Automatica, 50(9):2254-2268, 2014.

[17] S. Devasia. Should model-based inverse inputs be used as feedforward under plant uncertainty? IEEE Transactions on Automatic Control, 47(11):1865-1871, Nov, 2002.

[18] R. C. Dorf and R. H. Bishop. Modern Control Systems, 8th Edition. Addison Wesley, 1998. 